the UV irradiance in Kaitaia. Given that other factors such as diet and medical treatment were not significantly different between the two populations, the substantially earlier onset of age-related cataract in NZ dogs is highly likely to be related to high UV levels associated with depletion in the ozone layer at southern latitudes.

\section{Survey of owner attitudes toward the application of eye drops in dogs and cats with ophthalmic disease}

\author{
Sophie Mead, David Williams \\ University of Cambridge, Cambridge, United Kingdom
}

\section{OBJECTIVES}

Compliance of veterinary clients in administering ophthalmic medication to their pets has received little attention compared to compliance in human medicine. This study aimed to investigate owner attitudes regarding the ease of eyedrop administration.

\section{METHODS}

This study used questionnaire-guided interviews, asking what the temperament and ease of handling of the pet was, how often owners successfully applied drops, how easy they found it to apply medication (ease score: 1 very easy to 7 not possible), whether they needed help in providing treatment, whether the temperament of their pet had changed during treatment and if so in what manner, whether they had developed techniques to render treatment less stressful and how they felt about treating their pet. A convenience sampling method was used. All respondents gave informed consent to their opinions being evaluated.

\section{RESULTS}

Results from 15 cat owners and 33 dog owners showed an ease score for dogs was $3.3 \pm 0.4$ and for cats $4.5 \pm$ 0.4 and that temperament was not a predictor of ease of treatment. More compliant owners had more success treating their pets. $64 \%$ of dog owners and $93 \%$ of cat owners reported negative behavioural changes during treatment, including avoidance, nervousness and aggression. $58 \%$ of dog owners and $80 \%$ of cat owners associated treating their pets with negative emotions such as stress, worry, nervousness and guilt.

\section{STATEMENT (CONCLUSIONS)}

This study suggests that emotional factors and the impact of the client's relationship with their pet are important factors in compliance to prescribed treatment protocols.

\section{Evaluation of the I-PEN, a hand-held device for rapid measurement of tear osmolarity in the canine patient}

\section{Yuvani Bandara, David Williams}

Queen's Veterinary School Hospital, Cambridge, United Kingdom

\section{OBJECTIVES}

Previous reports have documented the use of electronic devices to measure tear-film osmolarity but devices such as the TearLab osmometer are cumbersome and expensive to use. Here a handheld solid state electronic diagnostic device for the quantitative measurement of tear-film osmolarity is evaluated for use in canine patients. The disposable tip of the device is held adjacent to the conjunctiva of the lower conjunctival sac and measures the impedance of the tear film bathing this tissue, calculating tear osmolarity within a few seconds.

\section{METHODS}

Animals assessed included twenty dogs with a normal tear film as diagnosed by ophthalmic examination and a Schirmer tear test (STT) reading over $15 \mathrm{~mm} / \mathrm{min}$, and ten dogs with keratoconjunctivitis sicca (KCS), diagnosed by finding of ocular surface pathology characteristic of dry eye and a STT reading of less than $15 \mathrm{~mm} / \mathrm{min}$.

\section{RESULTS}

The I-PEN device was easy to use and tolerated well by all animals. Osmolarity was $331.9 \pm 11.4 \mathrm{mOsm} / \mathrm{L}$ in normal eyes and $348.9 \pm 18.4 \mathrm{mOsm} / \mathrm{L}$ in eyes with KCS, these values being significantly different from each other $(p<0,05)$.

\section{STATEMENT (CONCLUSIONS)}

This study shows that the IPEN is a useful device to determine tear-film osmolarity in canine patients. Given that high osmolarity is thought to be a key factor in ocular surface pathology in dry eye, determination of tear osmolarity may be valuable in assessment of dogs with KCS 